PROCEEDINGS OF THE

AMERICAN MATHEMATICAL SOCIETY

Volume 128, Number 4, Pages 1003-1009

S 0002-9939(99)05469-6

Article electronically published on November 23, 1999

\title{
SEMI-CLASSICAL LIMIT OF WAVE FUNCTIONS
}

\author{
A. TRUMAN AND H. Z. ZHAO \\ (Communicated by James Glimm)
}

\begin{abstract}
We study in one dimension the semi-classical limit of the exact eigenfunction $\Psi_{E(N, h)}^{h}$ of the Hamiltonian $H=-\frac{1}{2} h^{2} \Delta+V(x)$, for a potential $V$ being analytic, bounded below and $\lim _{|x| \rightarrow \infty} V(x)=+\infty$. The main result of this paper is that, for any given $E>\min _{x \in R^{1}} V(x)$ with two turning points, the exact $L^{2}$ normalized eigenfunction $\left|\Psi_{E(N, h)}^{h}(q)\right|^{2}$ converges to the classical probability density, and the momentum distribution $\left|\hat{\Psi}_{E(N, h)}^{h}(p)\right|^{2}$ converges to the classical momentum density in the sense of distribution, as $h \rightarrow 0$ and $N \rightarrow \infty$ with $\left(N+\frac{1}{2}\right) h=\frac{1}{\pi} \int_{V(x)<E} \sqrt{2(E-V(x))} d x$ fixed. In this paper we only consider the harmonic oscillator Hamiltonian. By studying the semi-classical limit of the Wigner's quasi-probability density and using the generating function of the Laguerre polynomials, we give a complete mathematical proof of the Correspondence Principle.
\end{abstract}

\section{INTRODUCTION}

Consider the Schrödinger operator in $R^{1}$

$$
H=-\frac{1}{2} h^{2} \Delta+V(x)
$$

with $V(x)$ analytic, bounded below and $\lim _{|x| \rightarrow \infty} V(x)=+\infty$. Then by the limit point criteria, $H$ is a self-adjoint operator with discrete spectrum $\{E(N, h)\}_{N=0,1, \ldots \text {, }}$ $E(N, h) \rightarrow+\infty$ as $N \rightarrow \infty$ with corresponding orthonormal eigenfunctions $\Psi_{E(N, h)}^{h}$ $\in L^{2}$ for any fixed $h>0$ (see [6]). It is a basic question to ask what is the semiclassical limit of $\left|\Psi_{E(N, h)}^{h}(q)\right|^{2}$ as $h \rightarrow 0, N \rightarrow+\infty$ ([5], 2], [1], [4]). We consider the small $h$ and high quantum number limit of $\left|\Psi_{E(N, h)}^{h}(q)\right|^{2}$ as $h \rightarrow 0, N \rightarrow \infty$ but with $\left(N+\frac{1}{2}\right) h$ remaining a constant, namely

$$
\left(N+\frac{1}{2}\right) h=F(E)=\frac{1}{\pi} \int_{V(x)<E} \sqrt{2(E-V(x))} d x
$$

which means $E(N, h) \sim E$ for an arbitrary constant $E>\min V(x)$. For the momentum distribution, we consider

$$
\hat{\Psi}_{E(N, h)}^{h}(p)=(2 \pi h)^{-\frac{1}{2}} \int \mathrm{e}^{-\frac{i}{h} p q} \Psi_{E(N, h)}^{h}(q) d q,
$$

Received by the editors April 9, 1998.

2000 Mathematics Subject Classification. Primary 35Q40; Secondary 81Q20.

Key words and phrases. Schrödinger operator, semi-classical limit, weak convergence, probability density.

The research is supported by the EPSRC grants GR/L37823 and GR/K70397. 
and what is the semi-classical limit of $\left|\hat{\Psi}_{E(N, h)}^{h}(p)\right|^{2}$ as $h \rightarrow 0, N \rightarrow \infty$ but with $\left(N+\frac{1}{2}\right) h=F(E)$ ?

For an $L^{2}$ eigenfunction $\Psi$ of the Hamiltonian $H$, define Wigner's quasiprobability density by

$$
W_{\Psi}^{h}(q, p)=\frac{1}{2 \pi} \int \mathrm{e}^{-i \eta p} \Psi^{*}\left(q-\frac{1}{2} h \eta\right) \Psi\left(q+\frac{1}{2} h \eta\right) d \eta .
$$

Then it is well known that

$$
\int W_{\Psi}^{h}(q, p) d p=|\Psi(q)|^{2}, \quad \int W_{\Psi}^{h}(q, p) d q=|\hat{\Psi}(p)|^{2} .
$$

$W_{\Psi}^{h}$ is the quantum mechanical analogue of a simultaneous probability distribution. Of course, because $[q, p]=i h$, such a distribution can only exist in the limit as $h \rightarrow 0$. This is reflected in the fact that if $h>0$, in general

$$
W_{\Psi}^{h}(q, p) \nsupseteq 0 .
$$

It is straightforward to show that

$$
\begin{aligned}
\mathcal{F} W_{\Psi}^{h}(\beta, \alpha) & =\int \mathrm{e}^{i(\alpha p+\beta q)} W_{\Psi}^{h}(q, p) d q d p \\
& =\frac{1}{2 \pi} \int \mathrm{e}^{i(-\eta p+\alpha p+\beta q)} \Psi^{*}\left(q-\frac{1}{2} h \eta\right) \Psi\left(q+\frac{1}{2} h \eta\right) d \eta d q d p \\
& =\int \delta(\eta-\alpha) \mathrm{e}^{i \beta q} \Psi^{*}\left(q-\frac{1}{2} h \eta\right) \Psi\left(q+\frac{1}{2} h \eta\right) d \eta d q \\
& =\int \mathrm{e}^{i \beta q} \Psi^{*}\left(q-\frac{1}{2} h \alpha\right) \Psi\left(q+\frac{1}{2} h \alpha\right) d q .
\end{aligned}
$$

In order to study the semi-classical limits of $\left|\Psi_{E(N, h)}^{h}(q)\right|^{2}$ and $\left|\hat{\Psi}_{E(N, h)}^{h}(p)\right|^{2}$, by (4), we consider the corresponding Wigner quasi-probability density in the limit as $h \rightarrow 0, N \rightarrow \infty$ with $\left(N+\frac{1}{2}\right) h=F(E)$. To shed light on this question, in Section 2, we consider the harmonic oscillator Hamiltonian in detail. Its eigenvalues and eigenfunctions are well known. Here we give a complete mathematical proof of the Correspondence Principle in quantum mechanics (see [4, 2]). Some of the ideas for the proof appeared very briefly in [8]. We don't study the general case in this paper as the proof is lengthy and completely different. For this we need to use the WKB-Langer asymptotic solution. The key is our recent progress on solving a long standing problem that the WKB-Langer semi-classical solution is close to the exact $L^{2}$ eigenfunction in $L^{2}([9])$. We will publish the proof of the general case in our future publication.

In Section 3, we give some numerical simulations to illustrate the semi-classical limit results.

\section{HARMONIC OSCILlator HAMILTONIAN}

Consider the harmonic oscillator Hamiltonian

$$
H=-\frac{1}{2} h^{2} \Delta+\frac{1}{2} x^{2} .
$$

Recall that the $N$-th exact eigenfunction of the harmonic oscillator Hamiltonian (7) associated with the $N$-th eigenvalue $E(N, h)=\left(N+\frac{1}{2}\right) h$ is given by

$$
\Psi_{E(N, h)}^{h}(x)=A_{N} \mathcal{H}_{N}\left(\frac{x}{\sqrt{h}}\right) \mathrm{e}^{-\frac{x^{2}}{2 h}}, N=0,1,2, \cdots,
$$


and $\mathcal{H}_{N}$ is the $N$-th order Hermite polynomial $\mathcal{H}_{N}(\xi)=\mathrm{e}^{\xi^{2}}\left(-\frac{d}{d \xi}\right)^{N} \mathrm{e}^{-\xi^{2}}$, where $A_{N}=\left(2^{N} N ! \sqrt{\pi h}\right)^{-\frac{1}{2}}$ are the normalization constants.

Let $\mathcal{S}$ be the Schwartz space of rapidly decreasing functions in $R^{1}$ and $\mathcal{S}^{\prime}$ be the space of tempered distributions with weak topology. Then it is well known that $\Psi_{E(N, h)}^{h} \in \mathcal{S}$ for any $N$ and $h$.

It is not obvious at all what the limit of $\Psi_{E(N, h)}^{h}$ is as $h \rightarrow 0, N \rightarrow+\infty$ when $N h$ remains a constant. To investigate this, we consider the Fourier transform of Wigner's quasi-probability density.

Lemma 1. Let $\Psi_{E(N, h)}^{h}$ be the $N$-th eigenfunction of (7). Then

$$
\mathcal{F} W_{\Psi_{E(N, h)}^{h}}^{h}(\beta, \alpha)=\exp \left\{-\frac{|\theta|^{2}}{4}\right\} L_{N}^{0}\left(\frac{|\theta|^{2}}{2}\right),
$$

where $\theta=h^{\frac{1}{2}} \beta+i h^{\frac{1}{2}} \alpha$ and $L_{N}^{0}$ is the $N$-th Laguerre polynomial

$$
L_{N}^{0}(z)=\mathrm{e}^{z}\left(\frac{d}{d z}\right)^{N}\left(\mathrm{e}^{-z} z^{N}\right), N=0,1,2, \cdots .
$$

Proof. Substituting (8) into (6), we obtain

$$
\begin{aligned}
& \mathcal{F} W_{\Psi_{E(N, h)}^{h}}^{h}(\beta, \alpha) \\
= & \frac{1}{2^{N} N ! \sqrt{\pi h}} \int \mathrm{e}^{i \beta q} \mathcal{H}_{N}\left(\frac{q-\frac{1}{2} h \alpha}{\sqrt{h}}\right) \mathrm{e}^{-\frac{\left(q-\frac{1}{2} h \alpha\right)^{2}}{2 h}} \mathcal{H}_{N}\left(\frac{q+\frac{1}{2} h \alpha}{\sqrt{h}}\right) \mathrm{e}^{-\frac{\left(q+\frac{1}{2} h \alpha\right)^{2}}{2 h}} d q .
\end{aligned}
$$

Set

$$
\begin{aligned}
F(t) & =\sum_{N=0}^{\infty} t^{N} \mathcal{F} W_{\Psi_{E(N, h)}^{h}}^{h}(\beta, \alpha) \\
& =\sum_{N=0}^{\infty} \frac{t^{N}}{2^{N} N ! \sqrt{\pi h}} \int \mathrm{e}^{i \beta q-\frac{1}{h}\left(q^{2}+\frac{1}{4} h^{2} \alpha^{2}\right)} \mathcal{H}_{N}\left(\frac{q-\frac{1}{2} h \alpha}{\sqrt{h}}\right) \mathcal{H}_{N}\left(\frac{q+\frac{1}{2} h \alpha}{\sqrt{h}}\right) d q .
\end{aligned}
$$

But from the Mehler's kernel formula for $|t|<1,(x, y) \in R^{2}$,

$$
\begin{aligned}
& \sum_{N=0}^{\infty} \frac{t^{N}}{2^{N} N ! \sqrt{\pi}} \mathcal{H}_{N}\left(\frac{x}{\sqrt{h}}\right) \mathcal{H}_{N}\left(\frac{y}{\sqrt{h}}\right) \mathrm{e}^{-\frac{\left(x^{2}+y^{2}\right)}{2 h}} \\
= & \left(\pi h\left(1-t^{2}\right)\right)^{-\frac{1}{2}} \exp \left\{\frac{4 x y t-\left(1+t^{2}\right)\left(x^{2}+y^{2}\right)}{2\left(1-t^{2}\right) h}\right\} .
\end{aligned}
$$

Changing the order of summation and integration in (11) and using (12), we obtain

$$
\begin{aligned}
F(t) & =\left(\pi h\left(1-t^{2}\right)\right)^{-\frac{1}{2}} \int \exp \left\{i \beta q+\frac{4\left(q^{2}-\frac{1}{4} h^{2} \alpha^{2}\right) t-\left(1+t^{2}\right)\left(2 q^{2}+\frac{1}{2} h^{2} \alpha^{2}\right)}{2\left(1-t^{2}\right) h}\right\} d q \\
& =\left(\pi h\left(1-t^{2}\right)\right)^{-\frac{1}{2}} \int \exp \left\{i \beta q-\frac{2(1-t)}{(1+t) h} \frac{q^{2}}{2}-\frac{1}{4} h \alpha^{2} \frac{1+t}{1-t}\right\} d q \\
& =\frac{(1+t)^{\frac{1}{2}}}{(1-t)^{\frac{1}{2}}}\left(1-t^{2}\right)^{-\frac{1}{2}} \exp \left\{-\frac{1}{4} h \beta^{2} \frac{1+t}{1-t}-\frac{1}{4} h \alpha^{2} \frac{1+t}{1-t}\right\} \\
& =\frac{1}{1-t} \exp \left\{-\frac{1}{4}\left(1+\frac{2 t}{1-t}\right)\left(h \beta^{2}+h \alpha^{2}\right)\right\} .
\end{aligned}
$$


This gives

$$
F(t)=\frac{1}{1-t} \exp \left\{-\frac{z t}{1-t}\right\} \exp \left\{-\frac{1}{4}\left(h \beta^{2}+h \alpha^{2}\right)\right\}
$$

Here $z=\frac{|\theta|^{2}}{2}=\frac{1}{2}\left(h \alpha^{2}+h \beta^{2}\right)$ and $\theta=h^{\frac{1}{2}} \beta+i h^{\frac{1}{2}} \alpha$. Since $\frac{1}{1-t} \exp \left\{-\frac{z t}{1-t}\right\}$ is the generating function for Laguerre polynomials, comparing this and (13), we obtain (9).

Note that $\exp \left\{-\frac{|\theta|^{2}}{4}\right\} L_{N}^{0}\left(\frac{|\theta|^{2}}{2}\right)$ in (9) is in Schwartz space $\mathcal{S}$.

Lemma 2. For any given $E>0$, and each $(q, p) \in R^{2}$, the Wigner's quasiprobability density of the harmonic oscillator Hamiltonian $H$ has the semi-classical limit

$$
\lim _{\substack{N \rightarrow+\infty \\ h \rightarrow 0 \\ N h=E}} W_{\Psi_{E(N, h)}^{h}}^{h}(q, p)=\frac{1}{2 \pi} \delta\left(\frac{p^{2}}{2}+\frac{q^{2}}{2}-E\right)
$$

in $\mathcal{S}^{\prime}$.

Proof. We use the well-known result that, for $\nu=0,1,2, \cdots$,

$$
\lim _{N \rightarrow \infty} N^{-\nu} L_{N}^{\nu}\left(\frac{z}{N}\right)=z^{-\frac{\nu}{2}} J_{\nu}\left(2 z^{\frac{1}{2}}\right)
$$

uniformly for bounded $z$ ([7]). We set $\nu=0$ and $z=E\left(\beta^{2}+\alpha^{2}\right) / 2$ and use Bessel's integral representation for $J_{0}$, for each $(\beta, \alpha) \in R^{2}$

$$
\lim _{\substack{N \rightarrow+\infty \\ h \rightarrow 0 \\ N h=E}} \mathcal{F} W_{\Psi_{E(N, h)}^{h}}^{h}(\beta, \alpha)=\lim _{N \rightarrow \infty} L_{N}^{0}\left(\frac{E\left(\beta^{2}+\alpha^{2}\right)}{2 N}\right)=J_{0}\left(\sqrt{2 E\left(\beta^{2}+\alpha^{2}\right)}\right),
$$

which is from Bessel's integral ([10, p. 357])

$$
\lim _{\substack{N \rightarrow+\infty \\ h \rightarrow 0 \\ N h=E}} \mathcal{F} W_{\Psi_{E(N, h)}^{h}}^{h}(\beta, \alpha)=\frac{1}{\pi} \int_{0}^{\pi} \cos \left(\sqrt{2 E\left(\beta^{2}+\alpha^{2}\right)} \sin \theta\right) d \theta .
$$

First observe that

$$
\begin{aligned}
\int \mathrm{e}^{i(\alpha p+\beta q)} \delta\left(\frac{p^{2}}{2}+\frac{q^{2}}{2}-E\right) d q d p & =\int \mathrm{e}^{i(\beta, \alpha)(q, p)} \delta\left(\frac{p^{2}}{2}+\frac{q^{2}}{2}-E\right) d q d p \\
& =\mathcal{F}\left(\delta\left(\frac{p^{2}}{2}+\frac{q^{2}}{2}-E\right)\right)(\beta, \alpha) \\
& =\int \mathrm{e}^{i \sqrt{\beta^{2}+\alpha^{2}} \sqrt{q^{2}+p^{2}} \sin \theta} \delta\left(\frac{p^{2}}{2}+\frac{q^{2}}{2}-E\right) d q d p .
\end{aligned}
$$


Here $\left(\frac{\pi}{2}-\theta\right)$ is the angle between $(\beta, \alpha)$ and $(q, p)$. Setting $r^{2}=p^{2}+q^{2}$ and changing the integral to polar coordinates give

$$
\begin{aligned}
& \mathcal{F}\left(\delta\left(\frac{p^{2}}{2}+\frac{q^{2}}{2}-E\right)\right)(\beta, \alpha)=\int_{0}^{\infty} \int_{0}^{2 \pi} r \mathrm{e}^{i r \sqrt{\beta^{2}+\alpha^{2}}} \sin \theta \delta\left(\frac{r^{2}}{2}-E\right) d \theta d r \\
& =\int_{0}^{2 \pi} \int_{0}^{\infty} \mathrm{e}^{i r \sqrt{\beta^{2}+\alpha^{2}}} \sin \theta \delta\left(\frac{r^{2}}{2}-E\right) d\left(\frac{r^{2}}{2}\right) d \theta \\
& =\int_{0}^{2 \pi} \mathrm{e}^{i \sqrt{2 E} \sqrt{\beta^{2}+\alpha^{2}} \sin \theta} d \theta \\
& =2 \int_{0}^{\pi} \cos \left\{\sqrt{2 E} \sqrt{\beta^{2}+\alpha^{2}} \sin \theta\right\} d \theta \\
& =2 \pi \lim _{\substack{N \rightarrow+\infty \\
h \rightarrow \rightarrow 0 \\
N h=E}} \mathcal{F} W_{\Psi_{E(N, h)}^{h}}^{h}(\beta, \alpha),
\end{aligned}
$$

pointwise. Then taking the inverse Fourier transform $\mathcal{F}^{*}$,

$$
\begin{aligned}
W_{\Psi_{E(N, h)}^{h}}^{h}(q, p) & =\mathcal{F}^{*}\left(\mathcal{F} W_{\Psi_{E(N, h)}^{h}}^{h}\right)(q, p) \\
& \rightarrow \frac{1}{2 \pi} \mathcal{F}^{*}\left(\mathcal{F}\left(\delta\left(\frac{p^{2}}{2}+\frac{q^{2}}{2}-E\right)\right)(\beta, \alpha)\right) \\
& =\frac{1}{2 \pi} \delta\left(\frac{p^{2}}{2}+\frac{q^{2}}{2}-E\right)(\beta, \alpha),
\end{aligned}
$$

in $\mathcal{S}^{\prime}$. The above convergence follows from the fact that $\mathcal{F}^{*}: \mathcal{S}^{\prime} \rightarrow \mathcal{S}^{\prime}$ is continuous with weak topology in $\mathcal{S}^{\prime}$.

The following theorem is the main result of this section.

Theorem 3. For any given $E>0$, the exact eigenfunction (8) and the corresponding momentum wave function have the following semi-classical limits:

$$
\lim _{\substack{N \rightarrow+\infty \\ h \rightarrow 0 \\ N h=E}}\left|\Psi_{E(N, h)}^{h}(q)\right|^{2}=\frac{1}{\pi \sqrt{2 E-q^{2}}} \chi_{\frac{1}{2} q^{2}<E}
$$

and

$$
\lim _{\substack{N \rightarrow+\infty \\ h \rightarrow 0 \\ N h=E}}\left|\hat{\Psi}_{E(N, h)}^{h}(p)\right|^{2}=\frac{1}{\pi \sqrt{2 E-p^{2}}} \chi_{\frac{1}{2} p^{2}<E},
$$

in $\mathcal{S}^{\prime}$

Proof. From Lemma 2 for each $\lambda \in R$,

$$
\begin{aligned}
& \lim _{\substack{h \rightarrow+\infty \\
h \rightarrow 0 \\
N h=E}} \int \mathrm{e}^{i \lambda p} W_{\Psi_{E(N, h)}^{h}}^{h}(q, p) d p=\frac{1}{2 \pi} \int \mathrm{e}^{i \lambda p} \delta\left(\frac{1}{2} p^{2}+\frac{1}{2} q^{2}-E\right) d p \\
& \quad=\frac{1}{2 \pi} \int \frac{1}{p} \mathrm{e}^{i \lambda p} \delta\left(\frac{1}{2} p^{2}+\frac{1}{2} q^{2}-E\right) d\left(\frac{1}{2} p^{2}\right) \\
& \quad=\frac{1}{2 \pi} \frac{1}{\sqrt{2 E-q^{2}}}\left(\mathrm{e}^{i \lambda \sqrt{2 E-q^{2}}}+\mathrm{e}^{-i \lambda \sqrt{2 E-q^{2}}}\right) \chi_{\frac{1}{2} q^{2}<E}
\end{aligned}
$$




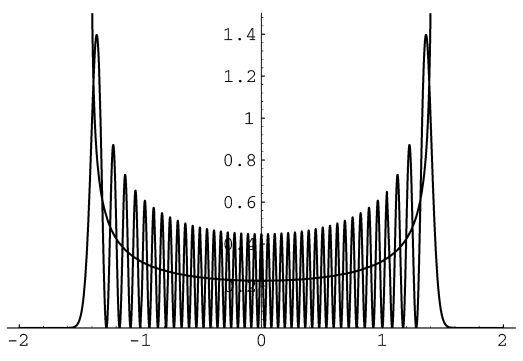

FiguRE 1. Probability densities for finding a quantum particle and a classical particle

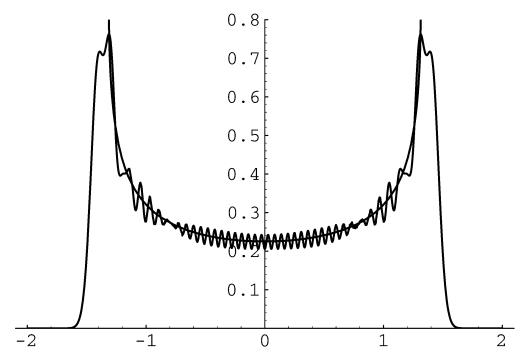

Figure 2. Semi-classical limit of the wave function in the sense of distribution, convergence of the integration

in $\mathcal{S}^{\prime}$. Set $\lambda=0$; then

$$
\lim _{\substack{h \rightarrow+\infty \\ h \rightarrow 0 \\ N h=E}}\left|\Psi_{E(N, h)}^{h}(q)\right|^{2}=\lim _{\substack{N \rightarrow+\infty \\ h \rightarrow 0 \\ N h=E}} \int W_{\Psi_{E(N, h)}^{h}}^{h}(q, p) d p=\frac{1}{\pi \sqrt{2 E-q^{2}}} \chi_{\frac{1}{2}} q^{2}<E,
$$

in $\mathcal{S}^{\prime}$. Similarly we can prove (17).

\section{NumERICAL SIMULATION}

In the last section, we demonstrate some simple numerical simulations of the semi-classical limit of the wave function produced by using Mathematica. We consider the harmonic oscillator studied analytically in Section 2. Here we take $N=40, h=1 / 40$ and $E=1$. In Figure 1, the quantum probability density $\left|\Psi_{N}^{h}(x)\right|^{2}$ is oscillatory and has $N$ zeros. The classical probability density curve which is nonoscillatory and nonzero lies in the center of the quantum curve. But the quantum curve does not approach the classical curve no matter how big an $N$ one uses. Therefore, we consider the integral

$$
I_{N, \epsilon}^{h}(x)=\frac{1}{2 \epsilon} \int_{x-\epsilon}^{x+\epsilon}\left|\Psi_{N}^{h}(y)\right|^{2} d y .
$$

However, as is shown in Figure 2, for $N=40, h=1 / 40, E=1, \epsilon=0.1$, the curve is very close to the corresponding classical one, i.e.

$$
J_{\epsilon}(x)=\frac{1}{2 \epsilon} \int_{x-\epsilon}^{x+\epsilon} \frac{1}{\pi \sqrt{2\left(E-\frac{1}{2} y^{2}\right)}} d y .
$$

This captures the essence of the convergence result in distribution sense obtained herein, i.e. $I_{N, \epsilon}^{h}(x) \rightarrow J_{\epsilon}(x)$ as $N \rightarrow \infty, h \rightarrow 0$ with $N h=E$.

\section{ACKNOWLEDGEMENT}

It is our pleasure to thank Professors K. D. Elworthy, D. Williams and W.A. Zheng for helpful conversations. One of us (AT) would like to thank Centre Emile Borel for hospitality during the Trimestre de Physique/Mathematique "Integrales Fonctonnelles", which enabled this paper to be finished. We would like to thank the referee for many very helpful comments. 


\section{REFERENCES}

[1] M.V. Berry, Waves near Stokes lines. I, Proc. R. Soc. Lond. A 427 (1990), 265-280. MR 91g:34027

[2] P.R. Holland, The quantum theory of motion. I, Cambridge University Press, Cambridge, 1993. MR 97e:81001

[3] R.E. Langer, On the connection formulas and the solutions of the wave equation. I, Physical Review 51 (1937), 669-676.

[4] R.L. Liboff, Introductory quantum mechanics. I, Addison-Wesley Publishing Company, 1980.

[5] V.P. Maslov and M.V. Fedoriuk, Semi-classical approximations in quantum mechanics. I, Reidel, Dordrecht, 1981. MR 84k:58226

[6] M. Reed and B. Simon, Methods of modern mathematical physics II: Fourier analysis, selfadjointness. I, Academic Press, New York, 1975. MR 58:12429b

[7] G. Szegoe, Orthogonal Polynomials. 3-rd edition. 1, Providence, 1967.

[8] M. Sirugue, M. Sirugue-Collin and A. Truman, Semi-classical approximation and microcanonical ensemble. I, Annales De L Institut Henri Poincare-Physique Theorique, 41 (1984), 429-444. MR 86g:81039

[9] A. Truman and H.Z. Zhao, WKB-Langer asymptotic expansions and $L^{2}$ convergence of eigenfunctions and their derivatives. I, Preprint (1998).

[10] E.T. Whittaker and G.N. Watson, Modern analysis, 2-nd edition. I, Cambridge University Press, Cambridge, 1915.

Department of Mathematics, University of Wales Swansea, Singleton Park, Swansea SA2 8PP, United Kingdom

E-mail address: A.Truman@swan.ac.uk

Current address, H. Z. Zhao: Department of Mathematical Sciences, Loughborough University, Loughborough LE11 3TU, United Kingdom

E-mail address: h.zhao@lboro.ac.uk 